\title{
Potential Candidates for a Structured Canadian ECPR Program for Out-of-Hospital Cardiac Arrest
}

\author{
Brian Grunau, MD* ${ }^{+\ddagger \S}$; Frank Xavier Scheuermeyer, MD, MHSc ${ }^{* \dagger}$; Dion Stub, MD, PhD* ${ }^{* 1 \|}$; \\ Robert H. Boone, MD, MSc***; Joseph Finkler, MD, MSc ${ }^{*{ }^{+}}$; Sarah Pennington, $\mathrm{RN}^{\dagger+}$; Sarah Ann

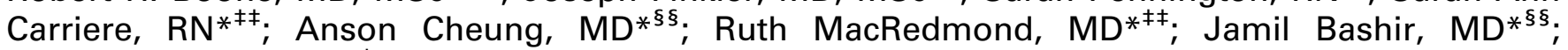 \\ Jim Christenson, $\mathrm{MD}^{* * * *}$
}

\section{ABSTRACT}

Objective: Extracorporeal cardiopulmonary resuscitation (ECPR), while resource-intensive, may improve outcomes in selected patients with refractory out-of-hospital cardiac arrest (OHCA). We sought to identify patients who fulfilled a set of ECPR criteria in order to estimate: (1) the proportion of patients with refractory cardiac arrest who may have benefited from ECPR; and (2) the outcomes achieved with conventional resuscitation.

Methods: We performed a secondary analysis from a 52-month prospective registry of consecutive adult nontraumatic OHCA cases from a single urban Canadian health region serving one million patients. We developed a hypothetical ECPR-eligible cohort including adult patients $<60$ years of age with a witnessed OHCA, and either bystander CPR or EMS arrival within five minutes. The primary outcome was the proportion of ECPR-eligible patients who had refractory cardiac arrest, defined as termination of resuscitation pre-hospital or in the ED. The secondary outcome was the proportion of EPCR-eligible patients who survived to hospital discharge.

Results: Of 1,644 EMS-treated OHCA, 168 (10.2\%) fulfilled our ECPR criteria. Overall, 54/1644 (3.3\%; 95\% Cl 2.4\%-4.1\%) who were ECPR-eligible had refractory cardiac arrest. Of ECPR-eligible patients, 114/168 (68\%, 95\% Cl 61\%-75\%) survived to hospital admission, and 70/168 (42\%; 95\% Cl $34-49 \%)$ survived to hospital discharge.

Conclusion: In our region, approximately $10 \%$ of EMS-treated cases of OHCA fulfilled our ECPR criteria, and approximately one-third of these (an average of 12 patients per year) were refractory to conventional resuscitation. The integration of an ECPR program into an existing high-performing system of care may have a small but clinically important effect on patient outcomes.

\section{RÉSUMÉ}

Objectif: La réanimation cardiorespiratoire extracorporelle (RCREC), bien qu'elle soit exigeante sur le plan des ressources, peut améliorer les résultats cliniques chez certains patients ayant subi un arrêt cardiaque extrahospitalier (ACEH) réfractaire. Les auteurs de l'étude cherchaient à déterminer les patients qui répondaient aux un ensemble de critères de la RCREC afin d'estimer, d'une part, la proportion de patients ayant subi un arrêt cardiaque réfractaire qui auraient pu profiter de la méthode de réanimation et, d'autre part, les résultats cliniques obtenus par la méthode habituelle de réanimation.

Méthode: Les auteurs ont procédé à une analyse secondaire d'un registre prospectif, constitué de cas consécutifs d'ACEH non traumatique, survenus chez des adultes, sur une période de 52 mois, dans une seule région sanitaire urbaine d'un million de personnes, au Canada. Les auteurs ont formé une cohorte fictive d'adultes, âgés de moins de 60 ans, susceptibles d'être traités par la RCREC après avoir subi un ACEH devant des témoins et avoir fait l'objet de manœuvres de réanimation cardiorespiratoire par des passants ou avoir reçu des soins donnés par des services médicaux d'urgence (SMU), et ce, en l'espace de cinq minutes. Le principal critère d'évaluation consistait en la proportion de patients susceptibles d'être traités par la RCREC après avoir subi un arrêt cardiaque réfractaire, défini comme la fin des manœuvres de réanimation avant l'arrivée à I'hôpital ou au service des urgences. Le critère d'évaluation secondaire était la proportion de patients susceptibles d'être traités par la RCREC qui ont survécu jusqu'au moment du congé de l'hôpital.

Résultats: Sur 1644 patients traités par les SMU pour un ACEH, $168(10,2 \%)$ répondaient aux critères de la RCREC et, dans 54 cas susceptibles d'être traités par la RCREC par rapport aux 1644 (3,3\%; IC à 95\% : 2,4\%-4,1\%), soit l'ensemble, il s'agissait d'un arrêt cardiaque réfractaire. Parmi les patients susceptibles d'être

From the *St. Paul's Hospital, Vancouver, BC; †Department of Emergency Medicine, University of British Columbia, Vancouver, BC; $¥$ Centre for Health Evaluation and Outcome Sciences, Vancouver, BC; §School of Population and Public Health, University of British Columbia, Vancouver, BC; IThe Alfred Hospital, Melbourne, Australia; IBBaker IDI Heart and Diabetes Institute, Melbourne, Australia; **Division of Cardiology, University of British Columbia, Vancouver, BC; ††Providence Healthcare Research Institute, Vancouver, BC; $¥ \neq D i v i s i o n$ of Critical Care, University of British Columbia, Vancouver, BC; and §§Division of Cardiovascular Surgery, University of British Columbia, Vancouver, BC.

Correspondence to: Dr. Brian Grunau, Emergency Department, St. Paul's Hospital, 1081 Burrard St., Vancouver, BC, Canada, V6Z $1 Y 6$. Email: Brian.Grunau2@vch.ca 
traités par la RCREC, 114/168 (68\%; IC à 95\% : 61\%-75\%) ont survécu jusqu'au moment de l'hospitalisation, et 70/168 (42\%; IC à 95\% : 34-49), jusqu'au moment du congé de I'hôpital.

Conclusions: Environ $10 \%$ de cas d'ACEH traités par les SMU dans la région répondaient aux critères de la RCREC et, dans environ un tiers d'entre eux (soit 12 patients par année, en moyenne), I'arrêt cardiaque était réfractaire aux manœuvres habituelles de réanimation cardiorespiratoire. L'intégration d'un programme de RCREC dans un système existant de soins à rendement élevé peut avoir un effet certes modeste mais important sur le plan clinique chez les patients.

Keywords: ECPR, ECMO, cardiopulmonary resuscitation, cardiac arrest

\section{INTRODUCTION}

In North America, emergency medical services (EMS) attend 134 cases of out-of-hospital cardiac arrest (OHCA) per 100,000 adult citizens annually, with survival ranging from $3 \%$ to $16 \% .{ }^{1,2}$ Circulatory support with extracorporeal cardiopulmonary resuscitation (ECPR) may improve the survival of select patients with cardiac arrest refractory to conventional resuscitation. ECPR is the incorporation of veno-arterial extracorporeal membranous oxygenation (ECMO) into cardiac arrest resuscitation. This technique has been in use since $1966 .^{3}$ Recent observational data suggests that ECPR may be a beneficial therapy for select patients with refractory OHCA, with most protocols focusing on younger patients with rapid arrest recognition and CPR initiation. ${ }^{4-7}$ Survival rates have been reported up to approximately $50 \% .^{5-9}$

Although ECPR may increase the proportion of survivors in selected OHCA patients, it is a resourceintensive therapy, requiring the attendance of a specially trained team that may include physicians, surgeons, cardiac perfusionists, and skilled nursing staff, as well as specialized protocols, equipment, and hospital resources. The aims of this descriptive study were: (1) to identify patients within our region who fulfilled a hypothetical set of ECPR criteria and estimate the number who would have been candidates for ECPR therapy; and (2) to determine the baseline outcomes of ECPR-eligible patients treated with conventional resuscitation to estimate the potential gains of an ECPR program.

\section{METHODS}

\section{Study setting}

This study took place in a single health region including the cities of Vancouver, Richmond, and North Vancouver, and the district municipalities of
North Vancouver and West Vancouver. The total land area is approximately $500 \mathrm{~km}^{2}$, and contains a population of approximately one million, with $73 \%$ between the ages of 15 and $65 .^{10}$

EMS are organized via a province-wide coordinated 911 service provided by the provincial British Columbia Ambulance Service (BCAS) as well as individual municipal fire departments. BCAS crews consist of two-person basic (BLS) or advanced (ALS) life support teams. ALS paramedics provide advanced cardiopulmonary life support (ACLS), ${ }^{11}$ including drug management and endotracheal intubation at the scene, while BLS paramedics provide basic life support resuscitation, including the use of automated external defibrillators (AEDs) and supraglottic airways. ${ }^{12}$ Fire department personnel are trained in cardiopulmonary resuscitation, ${ }^{12}$ including the use of AEDs. BCAS policy dictates which patients must be provided resuscitative treatments (see Appendix 1).

Four hospitals within the region receive patients with OHCA, all affiliated with the University of British Columbia. St. Paul's Hospital and Vancouver General Hospital have medical, cardiac surgery, and coronary critical care units, interventional cardiology capacity, electrophysiology services, and perform cardiac surgeries with perfusionist support. In addition, St. Paul's Hospital performs cardiac transplant and left ventricular assist device implantation procedures. Lion's Gate Hospital and Richmond General Hospital are community hospitals with intensive care and basic cardiology services but no catheterization laboratories. Although St. Paul's Hospital and Vancouver General Hospital provided ECMO services during the study period, there was no formal ECPR program either regionally or at any single institution.

The institutional review boards and affiliated ethics committees of Providence Health Care, the University of British Columbia, and Vancouver Coastal Health approved this study. 


\section{Selection of participants}

All consecutive non-traumatic OHCA patients within the region were prospectively identified as part of the Resuscitation Outcomes Consortium ${ }^{13}$ registry from September 2007 to December 2011. Based on previously published ECPR protocols, ${ }^{7,14,15}$ we constructed a post-hoc hypothetical cohort of "ECPReligible" patients from all EMS-treated cases of OHCA in the region with the following set of criteria: (1) age between 17 and 60 years; (2) witnessed arrest; and, (3) bystander CPR (performed by laypersons or EMS if the arrest was EMS-witnessed) or EMS arrival in less than five minutes. Patients who were known to have active malignancy were excluded from this cohort. Study patients who had termination of resuscitation in the pre-hospital or emergency department (ED) setting after full conventional efforts were considered to have refractory cardiac arrest and thereby were classified as potential candidates for ECPR therapy.

\section{Data collection}

We designed an electronic data collection tool with variables chosen a priori in consideration of Utstein variables, ${ }^{16}$ variables with known associations with outcomes in cardiac arrest, ${ }^{17}$ and variables to describe the patient population. All pre-hospital data, including time-stamped diagnostics, treatments administered, patient characteristics, and pre-hospital outcomes, were prospectively collected from standardized EMS template charting. ${ }^{13}$

Hospital-level data were abstracted through a systematic chart review, adhering to accepted criteria. ${ }^{18,19}$ We recorded the occurrence of invasive coronary angiography, coronary artery bypass surgery, targeted temperature management, and assessed outcomes at hospital discharge: (1) survival; and (2) neurologic outcome. Cases of missing or conflicting data were noted by each abstractor and prompted a full chart review; if still in question, the primary investigator reviewed the case. We selected 50 random charts via an electronic random number generator for a second independent chart review of outcome at hospital discharge. Cohen's kappa statistic was used to assess the inter-rater agreement for these 50 patients for the CPC score using a linear weighting scheme.

\section{Outcome measures and variable definitions}

The primary outcome was the proportion of patients in the region who were ECPR-eligible and had refractory cardiac arrest, defined as having resuscitative efforts terminated in the pre-hospital setting or the ED, prior to admission to a hospital critical care service. These patients represent the group of those with OHCA for whom ECPR may improve survival. The secondary outcomes were the proportion of ECPR-eligible patients who survived to hospital admission, hospital discharge, and the proportion of those with favourable neurological outcomes (defined as Cerebral Performance Category $[\mathrm{CPC}] 1-2^{20}$ ). All outcomes were also stratified by initial cardiac rhythm: (1) "shockable," including ventricular fibrillation, pulseless ventricular tachycardia, and unknown rhythms that were shocked with the AED; and (2) "non-shockable," including pulseless electrical activity, asystole, and unknown rhythms that were not shocked by the AED.

\section{Data analysis}

We used Microsoft Excel 2008 (Microsoft Corp., Redmond, WA, USA) for data entry and analysis. Descriptive statistics were used. Dichotomous variables are reported as percentages and $95 \%$ confidence intervals. Continuous variables are presented as means with standard deviations (if normally distributed) or medians with interquartile ranges (IQRs).

\section{RESULTS}

\section{Characteristics of study subjects}

In our health region between September 2007 and December 2011 (inclusive), there were 2,419 cases of OHCA, of whom 1,644 (68\%) were treated by EMS. Of EMS-treated cases, 168 (10.2\%) fulfilled our ECPR criteria (Figure 1). Inter-rater agreement for CPC score at hospital discharge was excellent with kappa values of $0.99(95 \% \mathrm{CI}=0.97-1.0)$.

\section{Main results}

Patient characteristics of the hypothetical ECPReligible cohort, and groups divided by initial rhythm, are shown in Table 1. There were 54 ECPR-eligible patients with refractory cardiac arrest in the region 


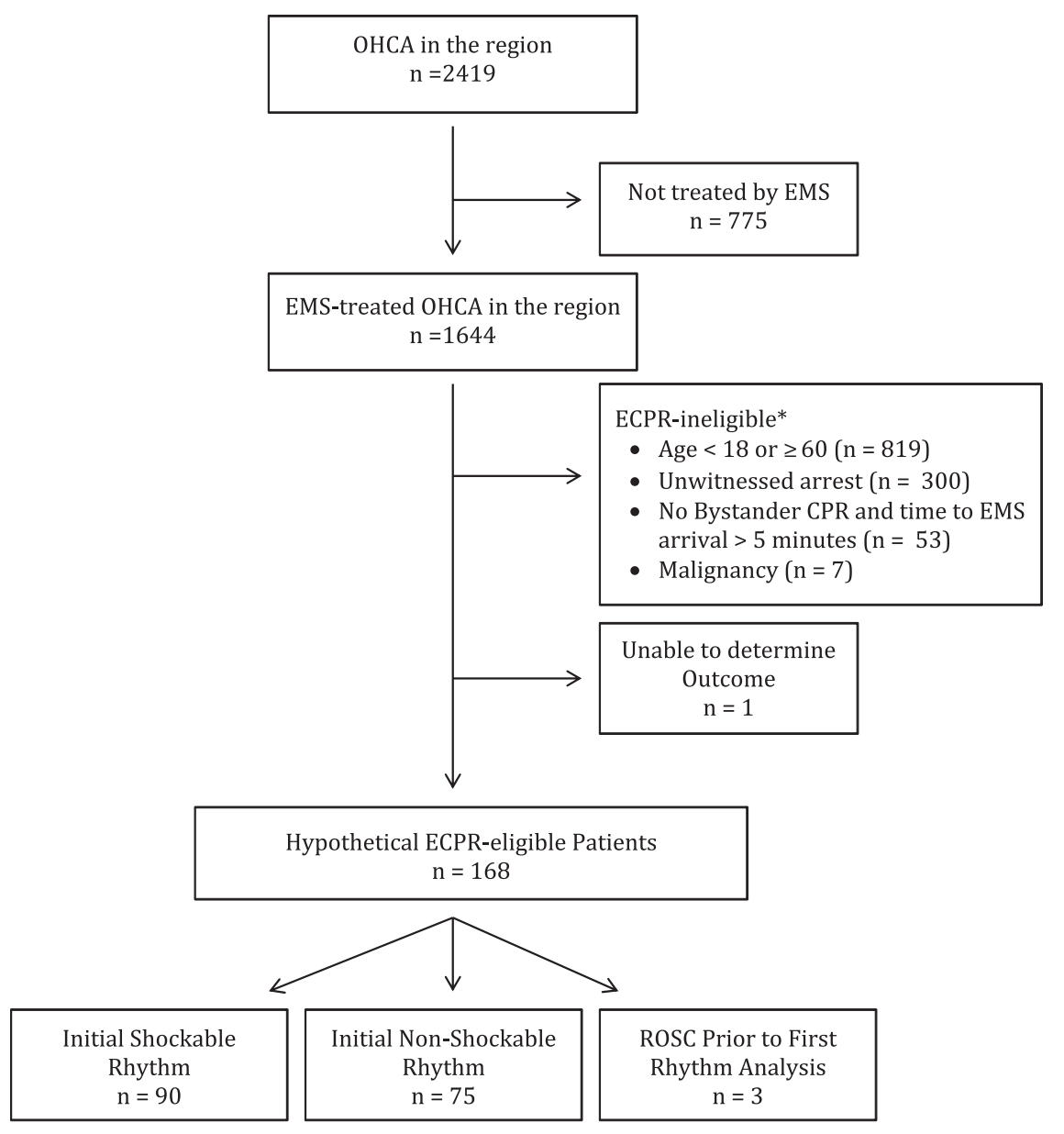

Figure 1. Study Flow

* Subjects excluded sequentially as per order listed.

(3.3\% of all EMS-treated cases, 95\% CI 2.4\%-4.1\%; $32 \%$ of all ECPR-eligible patients). Termination of resuscitation occurred in the pre-hospital setting in 30 cases $(56 \%)$ and in the ED in the other 24 (44\%), with a median duration of professional efforts of 38.4 minutes (IQR 33.6-46.3 minutes). Among the ECPR-eligible patients, $114(68 \%, 95 \%$ CI $61 \%-75 \%)$ survived to hospital admission, 70 (42\%; 95\% CI 34\%-49\%) survived to hospital discharge, and 59 (35\%; 95\% CI 28\%-42\%) had favourable neurological outcomes (Table 2).

Of the 165 patients for whom the initial cardiac rhythm was known, 90 patients (55\%) had initial shockable rhythms, of whom 78 (87\%) survived to hospital admission, 56 (62\%) survived to hospital discharge, and $49(54 \%)$ had favourable neurological outcomes (Table 2). For the 75 patients (45\%) with a nonshockable initial rhythm, 33 (44\%) survived to hospital admission, 11 (15\%) survived to hospital discharge, and seven $(9.3 \%)$ had favourable neurological outcomes.

\section{DISCUSSION}

We reported the characteristics and outcomes of patients within our region who fulfilled a hypothetical ECPR criteria, including young patients ( $<60$ yrs of age) with rapid CPR initiation. We then quantified the number of potential ECPR candidates who suffered from cardiac arrest refractory to conventional resuscitation. Within our regional population of approximately one million citizens, $3.3 \%$ of EMS-treated OHCA cases fulfilled our ECPR criteria and had refractory cardiac arrest, corresponding to an average of approximately one patient per month. Although patients in our ECPR-eligible cohort overall had a high proportion of positive outcomes, ${ }^{1}$ survival in this group may be enhanced with an ECPR program. These data can be used to estimate the resource burden and benefit an ECPR program may have in a region.

Several ECPR programs for OHCA have reported outcomes, with survival rates up to approximately $50 \%$ 


\begin{tabular}{|c|c|c|c|c|c|c|c|}
\hline & & \multicolumn{2}{|c|}{ "ECPR-Eligible" Cohort } & \multicolumn{2}{|c|}{ Initial Shockable Rhythms } & \multicolumn{2}{|c|}{ Initial Non-Shockable Rhythms } \\
\hline & & n (IQR or \%) & Missing & n (IQR or \%) & Missing & n (IQR or \%) & Missing \\
\hline \multirow[t]{3}{*}{ Demographics } & Number & 168 & & $90^{*}$ & & $75^{*}$ & \\
\hline & Age & $50(41-55)$ & 0 & $52(44-56)$ & 0 & $49(40-54)$ & 0 \\
\hline & Male & $131(78)$ & 0 & $74(82)$ & 0 & $57(76)$ & 0 \\
\hline \multirow[t]{13}{*}{$\mathrm{PMHx}$} & Myocardial infarction & $13(7.9)$ & 4 & $8(8.9)$ & 0 & $5(6.9)$ & 3 \\
\hline & Coronary artery disease & $17(10)$ & 4 & $10(11)$ & 0 & $7(9.7)$ & 3 \\
\hline & Hypertension & $38(23)$ & 4 & $25(28)$ & 0 & $13(18)$ & 3 \\
\hline & Congestive heart failure & $4(2.4)$ & 4 & $4(4.4)$ & 0 & 0 & 3 \\
\hline & Diabetes mellitus & $20(12)$ & 4 & $8(8.9)$ & 0 & $12(17)$ & 3 \\
\hline & Seizure & $3(1.8)$ & 4 & $1(1.1)$ & 0 & $2(2.8)$ & 3 \\
\hline & Syncope & $2(1.2)$ & 4 & $2(2.2)$ & 0 & 0 & 3 \\
\hline & Atrial fibrillation & $4(2.4)$ & 4 & $2(2.2)$ & 0 & $2(2.8)$ & 3 \\
\hline & Cardiac medications & $24(15)$ & 4 & $17(19)$ & 0 & $7(9.7)$ & 3 \\
\hline & Recreational drugs & $25(15)$ & 3 & $5(5.6)$ & 0 & $20(27)$ & 2 \\
\hline & Alcoholism & $24(15)$ & 3 & $8(8.9)$ & 0 & $16(22)$ & 2 \\
\hline & Pacemaker & $1(0.6)$ & 4 & $1(1.1)$ & 0 & 0 & 3 \\
\hline & Heart surgery & $5(3.0)$ & 4 & $1(1.1)$ & 0 & $4(5.6)$ & 3 \\
\hline \multirow[t]{15}{*}{ Cardiac Arrest } & Public location & $77(46)$ & 0 & $49(54)$ & 0 & $26(35)$ & 0 \\
\hline & Witnessed arrest & & & & & & \\
\hline & by Layperson & $133(79)$ & 0 & 77 (86) & 0 & $53(71)$ & 0 \\
\hline & by EMS & $35(21)$ & 0 & $13(14)$ & 0 & $22(29)$ & 0 \\
\hline & Bystander CPR & & & & & & \\
\hline & By Layperson & $114(68)$ & 0 & $67(74)$ & 0 & $44(59)$ & 0 \\
\hline & By EMS & $35(21)$ & 0 & $13(14)$ & 0 & $22(29)$ & 0 \\
\hline & Dispatch to EMS arrival (min) & $6.3(4.9-8.0)$ & 0 & $6.2(4.9-8.0)$ & 0 & $6.3(4.5-8.2)$ & 0 \\
\hline & ALS involvement & $165(98)$ & 0 & $88(98)$ & 0 & $74(99)$ & 0 \\
\hline & Initial arrest rhythm shockable & $90(54)$ & 3 & $90(100)$ & 0 & $0(0)$ & 0 \\
\hline & Any shocks delivered & $114(68)$ & 0 & $90(100)$ & 0 & $24(32)$ & 0 \\
\hline & Number of shocks & $2(1-4)$ & 0 & $2(1-5)$ & 0 & $2(1-3)$ & 0 \\
\hline & Epinephrine administered & $109(65)$ & 0 & $47(52)$ & 0 & $62(83)$ & 0 \\
\hline & Epinephrine dose (mg) & $5(2-7)$ & 0 & $4(2-6)$ & 0 & $5(3-7)$ & 0 \\
\hline & Transported to hospital & $138(82)$ & 0 & $88(98)$ & 0 & $47(63)$ & 0 \\
\hline \multirow[t]{3}{*}{ Hospital Care } & Coronary angiography & $59\left(52^{\dagger}\right)$ & 1 & $56\left(72^{\dagger}\right)$ & 1 & $3\left(9.1^{\dagger}\right)$ & 0 \\
\hline & CABG & $8\left(7.0^{\dagger}\right)$ & 1 & $8\left(10^{\dagger}\right)$ & 1 & 0 & 0 \\
\hline & MTH & $60\left(53^{\dagger}\right)$ & 1 & $52\left(67^{\dagger}\right)$ & 1 & $7\left(21^{\dagger}\right)$ & 0 \\
\hline
\end{tabular}

in select patients with persistent refractory cardiac arrest. $^{5-9}$ While no prospective randomized studies have been completed examining the outcomes of ECPRtreated patients in comparison to conventional resuscitation, ECPR for OHCA is typically initiated between 45 and 75 minutes after the initial cardiac arrest, ${ }^{6,7}$ an elapsed duration of resuscitation at which survival with conventional resuscitation appears to be rare. ${ }^{17}$ The Alfred Hospital and Victorian EMS system in Melbourne, Australia, which provides similar hospital and pre-hospital services to those in our region, recently described the results of an ECPR protocol, demonstrating 56\% neurologically intact survival in a carefully selected group of patients with refractory OHCA treated with ECPR. ${ }^{7}$ Based on these data and other similar studies, assuming a survival rate of $30 \%-55 \%$ of those in refractory cardiac arrest treated with ECPR, ${ }^{6-8}$ among the 54 potential ECPR candidates in our cohort who remained pulseless after conventional resuscitation, it is possible that an additional 16-30 patients (four 


\begin{tabular}{|c|c|c|c|}
\hline & $\begin{array}{l}\text { All Patients } \\
\text { n (\% or IQR) }\end{array}$ & $\begin{array}{c}\text { Initial Shockable Rhythms } \\
\text { n (\% or IQR) }\end{array}$ & $\begin{array}{c}\text { Initial Non-Shockable Rhythms } \\
\text { n (\% or IQR) }\end{array}$ \\
\hline Number & 168 & $90^{*}$ & $75^{*}$ \\
\hline ROSC & $129(77)$ & $84(93)$ & $42(56)$ \\
\hline Time to ROSC (minutes) & $11.9(6.3-20.8)$ & $10.8(6.1-20.5)$ & $15.0(7.2-22.3)$ \\
\hline Survival to Hospital Admission & $114(68,95 \% \mathrm{Cl} 61-75)$ & $78(87)$ & $33(44)$ \\
\hline Survival to Hospital Discharge & $70(42 ; 95 \% \mathrm{Cl} 34-49)$ & $56(62)$ & $11(15)$ \\
\hline CPC $1-2$ & 59 (35; 95\% Cl 28-42) & $49(54)$ & $7(9.3)$ \\
\hline
\end{tabular}

to seven per year) might have survived neurologically intact with an ECPR protocol, increasing the overall survival rate of ECPR-eligible patients to $51 \%-60 \%$.

Several studies have reported the number of patients treated with individual ECPR protocols, with patients included at the discretion of treating clinicians; ;,6,21-26 however, the denominator and outcomes of potentially eligible patients within these regions is unknown. Poppe and colleagues examined a cohort of OHCA cases, and applied the following criteria for which paramedics should "load [the patient] and go" to hospital for ECPR management: adult patients $<75$ years old with a witnessed arrest, bystander CPR performed, an initial shockable rhythm, and no sustained return of spontaneous circulation (ROSC) within 15 minutes of EMS resuscitation. ${ }^{27}$ Of 864 OHCA, 55 (6.4\%) met this standard. Despite methodological differences, this is comparable with our results in demonstrating a low proportion of patients with refractory arrest within our region who would be candidates for ECPR therapy.

The primary limiting factor of the quantity of eligible patients for ECPR is distance to hospital, with survivors rare if ECPR is initiated after 75 minutes. ${ }^{5,21,23}$ For this reason and based on established practice, ${ }^{7}$ we limited our hypothetical ECPR treatment catchment to our health region, for which the transport times for an ambulance to an ECPR-capable centre would be approximately 20 minutes or less. Although population density and demographics will vary, the time-dependent nature of ECPR therapy will likely limit the volume of ECPR programs in other regions to similar quantities.

Although some ECPR protocols have focused solely on patients with initial shockable rhythms, ${ }^{7,28}$ other protocols have shown promising results in nonshockable patients, reporting survival to discharge in $29 \%-35 \% 6,7,21$. It is unclear whether the benefit of
ECPR therapy for those with initial non-shockable rhythms differs from those with initial shockable rhythms. It is well established that patients with initial shockable rhythms treated with conventional resuscitation have a higher chance of achieving ROSC, thereby allowing the possibility of survival. ${ }^{1,29,30}$ However, in resuscitations unable to achieve ROSC, regardless of initial cardiac rhythm, the benefit of achieving artificial circulation through ECPR may be similar. Nearly $90 \%$ of the patients in our cohort with initial shockable rhythms survived to hospital admission, twice the proportion of those with initial non-shockable rhythms. Whereas patients with shockable rhythms fulfilling our ECPR criteria already demonstrate excellent outcomes, patients with initial non-shockable rhythms may in fact be the group for which an ECPR program may have a much larger impact on survival. One must also consider that there may systematic differences in patient characteristics between those with initial shockable and non-shockable rhythms, such as the etiologies of the arrest, which may affect outcomes. Further research is required to identify the best candidates for ECPR initiation and the outcomes of patients with nonshockable rhythms treated with ECPR.

The complexities of developing regional invasive OHCA resuscitation programs in systems with high existing rates of survival, ${ }^{1}$ with the hope of further improving outcomes, are extensive. Regional coordination, highly skilled paramedics, a strategy to maintain CPR quality during transport, physicians or surgeons to place cannulas and perfusionists to initiate ECMO immediately upon patient arrival, and sufficient patient volumes to maintain institutional competency are all important components. The creation and maintenance of such as system would require significant resources, and thus prospective studies (with comparator groups) examining the effectiveness and cost-effectiveness of 
coordinated regional ECPR programs are required; this is especially relevant for single-payer healthcare systems. Finally, over half of those with refractory cardiac arrest had termination of resuscitation in the pre-hospital setting. With the addition of ECPR management into the resuscitation algorithm, practices for intra-arrest transport may have to be adapted, including recognition of safety hazards for EMS providers during transport. ${ }^{31}$

The study site was one urban Canadian region; population characteristics and medical management may vary in different settings. Whereas pre-hospital resuscitation and protocolized hospital care followed AHA guidelines, ${ }^{11,12,32}$ we cannot account for individual patient treatment. It is possible that characteristics not represented in our set of ECPR criteria may be of benefit in further defining the ideal ECPR candidate. There were three patients within our ECPR group who regained a pulse prior to the first rhythm analysis, precluding inclusion in the rhythm subgroup analysis, and thus resulting in a lower proportion of favorable outcomes in one or both groups.

\section{CONCLUSION}

In our region, $10 \%$ of EMS-treated patients fulfilled our set of ECPR criteria, one-third of whom had resuscitation terminated prior to hospital admission (an average of 12 patients per year) and may have benefited from ECPR. The addition of an ECPR program to a highperforming system of care may have a small but clinically important effect on patient outcomes.

Competing Interests: None declared.

\section{SUPPLEMENTARY MATERIAL}

To view supplementary material for this article, please visit http://dx.doi.org/10.1017/cem.2016.8

\section{REFERENCES}

1. Nichol G, Thomas E, Callaway CW, et al. Regional variation in out-of-hospital cardiac arrest incidence and outcome. ZAMA 2008;300(12):1423-31, doi:10.1001/jama.300.12.1423.

2. Go AS, Mozaffarian D, Roger VL, et al. Heart disease and stroke statistics-2014 update: a report from the American Heart Association. Circulation 2014;129(3):e28-292, doi:10.1161/01.cir.0000441139.02102.80.

3. Kennedy $\mathrm{JH}$. The role of assisted circulation in cardiac resuscitation. FAMA 1966;197(8):615-8.
4. Morimura N, Sakamoto T, Nagao K, et al. Extracorporeal cardiopulmonary resuscitation for out-of-hospital cardiac arrest: A review of the Japanese literature. Resuscitation 2011;82(1):10-4, doi:10.1016/j.resuscitation.2010.08.032.

5. Wang CH, Chou NK, Becker LB, et al. Improved outcome of extracorporeal cardiopulmonary resuscitation for out-ofhospital cardiac arrest-a comparison with that for extracorporeal rescue for in-hospital cardiac arrest. Resuscitation 2014;85(9):1219-24, doi:10.1016/j.resuscitation.2014.06.022.

6. Leick J, Liebetrau C, Szardien S, et al. Door-toimplantation time of extracorporeal life support systems predicts mortality in patients with out-of-hospital cardiac arrest. Clin Res Cardiol 2013;102(9):661-9, doi:10.1007/ s00392-013-0580-3.

7. Stub D, Bernard S, Pellegrino V, et al. Refractory cardiac arrest treated with mechanical CPR, hypothermia, ECMO and early reperfusion (the CHEER trial). Resuscitation 2015;86:88-94, doi:10.1016/j.resuscitation.2014.09.010.

8. Bellezzo JM, Shinar Z, Davis DP, et al. Emergency physician-initiated extracorporeal cardiopulmonary resuscitation. Resuscitation 2012;83(8):966-70, doi:10.1016/j. resuscitation.2012.01.027.

9. Siao FY, Chiu CC, Chiu CW, et al. Managing cardiac arrest with refractory ventricular fibrillation in the emergency department: Conventional cardiopulmonary resuscitation versus extracorporeal cardiopulmonary resuscitation. Resuscitation 2015;92:70-6, doi:10.1016/j.resuscitation.2015.04.016.

10. Statistics Canada. Focus on Geography Series, 2011 Census. Ottawa, Ontario, 2012.

11. Link MS, Berkow LC, Kudenchuk PJ, et al. Part 7: Adult Advanced Cardiovascular Life Support: 2015 American Heart Association Guidelines Update for Cardiopulmonary Resuscitation and Emergency Cardiovascular Care. Circulation 2015;132(18 Suppl 2):S444-64, doi:10.1161/ CIR.0000000000000261.

12. Kleinman ME, Brennan EE, Goldberger ZD, et al. Part 5: Adult Basic Life Support and Cardiopulmonary Resuscitation Quality: 2015 American Heart Association Guidelines Update for Cardiopulmonary Resuscitation and Emergency Cardiovascular Care. Circulation 2015;132(18 Suppl 2): S414-35, doi:10.1161/CIR.0000000000000259.

13. Morrison LJ, Nichol G, Rea TD, et al. Rationale, development and implementation of the Resuscitation Outcomes Consortium Epistry-Cardiac Arrest. Resuscitation 2008;78(2): 161-9, doi:10.1016/j.resuscitation.2008.02.020.

14. Fagnoul D, Taccone FS, Belhaj A, et al. Extracorporeal life support associated with hypothermia and normoxemia in refractory cardiac arrest. Resuscitation 2013;84(11):1519-24, doi:10.1016/j.resuscitation.2013.06.016.

15. Johnson NJ, Acker M, Hsu CH, et al. Extracorporeal life support as rescue strategy for out-of-hospital and emergency department cardiac arrest. Resuscitation 2014;85(11):1527-32, doi:10.1016/j.resuscitation.2014.08.028.

16. Jacobs I, Nadkarni V, Bahr J, et al. Cardiac arrest and cardiopulmonary resuscitation outcome reports: update and simplification of the Utstein templates for resuscitation registries. A statement for healthcare professionals from a task force of the international liaison committee on resuscitation (American Heart Association, European Resuscitation Council, Australian Resuscitation Council, New Zealand 
Resuscitation Council, Heart and Stroke Foundation of Canada, InterAmerican Heart Foundation, Resuscitation Council of Southern Africa). Resuscitation 2004;63(3):233-49, doi:10.1016/j.resuscitation.2004.09.008.

17. Reynolds JC, Frisch A, Rittenberger JC, et al. Duration of resuscitation efforts and functional outcome after out-ofhospital cardiac arrest: when should we change to novel therapies? Circulation 2013;128(23):2488-94, doi:10.1161/ CIRCULATIONAHA.113.002408.

18. Gilbert EH, Lowenstein SR, Koziol-McLain J, et al. Chart reviews in emergency medicine research: Where are the methods? Ann Emerg Med 1996;27(3):305-8.

19. Worster A, Bledsoe RD, Cleve P, et al. Reassessing the methods of medical record review studies in emergency medicine research. Ann Emerg Med 2005;45(4):448-51, doi:10.1016/j.annemergmed.2004.11.021.

20. Jacobs I, Nadkarni V, Bahr J, et al. Cardiac arrest and cardiopulmonary resuscitation outcome reports: update and simplification of the Utstein templates for resuscitation registries: a statement for healthcare professionals from a task force of the International Liaison Committee on Resuscitation (American Heart Association, European Resuscitation Council, Australian Resuscitation Council, New Zealand Resuscitation Council, Heart and Stroke Foundation of Canada, InterAmerican Heart Foundation, Resuscitation Councils of Southern Africa). Circulation 2004;110(21):3385-97, doi:10.1161/01.CIR.0000147236.85306.15.

21. Haneya A, Philipp A, Diez C, et al. A 5-year experience with cardiopulmonary resuscitation using extracorporeal life support in non-postcardiotomy patients with cardiac arrest. Resuscitation 2012;83(11):1331-7, doi:10.1016/j.resuscitation. 2012.07.009.

22. Avalli L, Maggioni E, Formica F, et al. Favourable survival of in-hospital compared to out-of-hospital refractory cardiac arrest patients treated with extracorporeal membrane oxygenation: an Italian tertiary care centre experience. Resuscitation 2012;83(5):579-83, doi:10.1016/j.resuscitation. 2011.10.013.

23. Le Guen M, Nicolas-Robin A, Carreira S, et al. Extracorporeal life support following out-of-hospital refractory cardiac arrest. Crit Care 2011;15(1):R29. doi:10.1186/cc9976.

24. Maekawa K, Tanno K, Hase $M$, et al. Extracorporeal cardiopulmonary resuscitation for patients with out-of- hospital cardiac arrest of cardiac origin: a propensitymatched study and predictor analysis. Crit Care Med 2013; 41(5):1186-96, doi:10.1097/CCM.0b013e31827ca4c8.

25. Nagao K, Kikushima K, Watanabe K, et al. Early induction of hypothermia during cardiac arrest improves neurological outcomes in patients with out-of-hospital cardiac arrest who undergo emergency cardiopulmonary bypass and percutaneous coronary intervention. Circ 7 2010;74(1):77-85, doi:10.1253/circj.CJ-09-0502.

26. Kim SJ, Jung JS, Park JH, et al. An optimal transition time to extracorporeal cardiopulmonary resuscitation for predicting good neurological outcome in patients with out-of-hospital cardiac arrest: a propensity-matched study. Crit Care 2014; 18(5):535. doi:10.1186/s13054-014-0535-8.

27. Poppe M, Schober A, Weiser C, et al. The incidence of "load\&go" out-of-hospital-cardiac arrest-candidates for emergency department utilization of emergencyextracorporeal life support. A one-year review. Resuscitation 2015;91:131-6, doi:10.1016/j.resuscitation.2015.03.003.

28. Sakamoto T, Morimura N, Nagao K, et al. Extracorporeal cardiopulmonary resuscitation versus conventional cardiopulmonary resuscitation in adults with out-of-hospital cardiac arrest: A prospective observational study. Resuscitation 2014; 85(6):762-8, doi:10.1016/j.resuscitation.2014.01.031.

29. Andrew E, Nehme Z, Lijovic M, et al. Outcomes following out-of-hospital cardiac arrest with an initial cardiac rhythm of asystole or pulseless electrical activity in Victoria, Australia. Resuscitation 2014;85(11):1633-9, doi:10.1016/j. resuscitation.2014.07.015.

30. Wibrandt I, Norsted K, Schmidt H, et al. Predictors for outcome among cardiac arrest patients: the importance of initial cardiac arrest rhythm versus time to return of spontaneous circulation, a retrospective cohort study. BMC Emerg Med 2015;15:3. doi:10.1186/s12873-015-0028-3.

31. Kahn CA, Pirrallo RG, Kuhn EM. Characteristics of fatal ambulance crashes in the United States: an 11-year retrospective analysis. Prehosp Emerg Care 2001;5(3):261-9.

32. Callaway CW, Donnino MW, Fink EL, et al. Part 8: Post-Cardiac Arrest Care: 2015 American Heart Association Guidelines Update for Cardiopulmonary Resuscitation and Emergency Cardiovascular Care. Circulation 2015; 132(18 Suppl 2):S465-82, doi:10.1161/CIR.0000000000000262. 